\title{
8b/10b Encoder Design
}

\author{
Xu Qiaoyu \\ School of Electromechanical Engineering \\ Henan University of Science and Technology \\ Luoyang, China
}

\author{
Liu Huijie \\ School of Electromechanical Engineering \\ Henan University of Science and Technology \\ Luoyang, China
}

\begin{abstract}
In order to resolve the problem of base-line offset and unbalanced code flow during the fiber data transmission, thesis give a simple and practical solution: $8 \mathrm{~B} / 10 \mathrm{~B}$ encode. This solution taking an method which integrate checking scheme and logic operation, through Verilog HDL description language, realize the design of encoder. After lots of simulation and application, the results indicate: this amout of computation is small, quick speed, high reliability, and can achieve $8 \mathrm{~B} / 10 \mathrm{~B}$ code function effectively.
\end{abstract}

Keywords-8B/10B code, Verilog HDL language,Quartus II9.0 software, Polarity deviation RD

\section{INTRODUCTION}

Due the quick development of communication of technology,fiber communication is more and more popular for people, at the same time, its higher transmission speed and bigger capacity are required by people. But, high-speed fiber transmission bring problem as base-line offset and unbalanced code flow. So, based on such problems, we design the $8 \mathrm{~B} / 10 \mathrm{~B}$ encode, because its low transmission mistake percent and DC compensation function, also with checking mistake fuction during the transmission and special function[1], it resolve the problem as base-line offset and unbalanced code flow, but also was used in high-speed sbus.

On the way to complete $8 \mathrm{~B} / 10 \mathrm{~B}$. In local, it was designed to save the prepared code into an Programmable Read-Only Memory, this asimple method, but limit the speed of read. In foreign, it was finished by integrated logical circuit, that's mean to integrate total encoder into a small chip, was composed into a big special integrated encoder, its advantage is simple circuit, but the disadvantage is too complicated logic[2].

\section{SOLUTION AND CONCEPT}

In order to finish the encoder function during the fiber data transmission, we design the concept, which have three function model: checking mistake and special code $、 8 \mathrm{~B} / 10 \mathrm{~B}$ encoder and FIFO. Disaplay in the Figure.1[3].

(1).Checking mistake and special code: For the input $8 \mathrm{~B}$, after checked mistake and special code, if have mistake or special code, it will output a symbol bit.

(2). $8 \mathrm{~B} / 10 \mathrm{~B}$ Encoder: transfer the input $8 \mathrm{~B}$ into $10 \mathrm{~B}$ as output, this function model is the core function of the concept.

(3).FIFO: FIFO data buffer.

\section{ENCODER PRINCIPL}

The principle of encoder is: mapping transfer the input 8 bits data into 10 bits data per the mapping principle, separate the 8 bits data into one group 3 bits data and another group 5 bits data, after encoding of the $8 \mathrm{~B} / 10 \mathrm{~B}$ encoder, output one group 4 bits data and another group 6 bits data, to make a 10 bite data, the situation of number 0 and number 1 together is three: 5 times number 0 and 5 times number $1 ; 4$ times number 0 and 6 times number $1 ; 6$ times number 0 and 4 times number 1 . Above three situation have three unbalance $0,-2,+2$ accordingly. During the process of encoding, mark this unbalance with an parameter :Polarity deviation RD . this polarity deviation $\mathrm{RD}$ is composed with $\mathrm{RD}-$ and $\mathrm{RD}+$. $\mathrm{RD}$ - means the times of number 1 is 2 times more than the times of number 0 ; RD+ means the times of number 0 is 2 times more than the times of number 1 [4].

The original 8B data was separated into two part: front 3 bits and back 5 bits. For front 3 bits, encoding it per $3 \mathrm{~B} / 4 \mathrm{~B}$; for the back 5bits, encoding it per $5 \mathrm{~B} / 6 \mathrm{~B}$. As show in Table.1. The order of original $8 \mathrm{~B}$ data is HGFEDCBA, HGF encoded into fghj; EDCBA encoded into abcdei. So, the transferred $10 \mathrm{~B}$ is abcdeifghj. After the transformation, the data flow was output by $10 \mathrm{~B}$. As show in Figure.2.

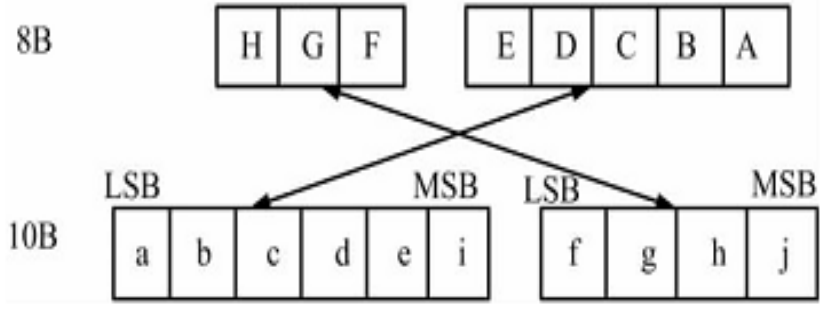

Figure 2. 8B/10B encoding relationship

It is important to ensure the DC balance of data flow during the transmission process, also need to think about the unbalance of DC data flow. As show in Table.2, for each 8B data, after encoding, it will be transferred into one kind of RD-\&RD+. Encoder will decide the next $10 \mathrm{~B}$ data based on previous RD and ensure the DC data flow balance .

The original status of encoder is RD-, it will check the data flow, if the quantity of number 1 and number 0 is the same, the polarity of next 10B data will keep RD-, otherwise, it will turn to be $\mathrm{RD}+$. If previous $10 \mathrm{~B}$ polarity is $\mathrm{RD}+$, and the quantity of number 1 and number 0 of previous 10B data keep same, the polarity of next $10 \mathrm{~B}$ data will keep $\mathrm{RD}+$, 
otherwise, it will turn to be RD-[5].

\section{8B/10B ENCODER DESIGN AND COMPLETE}

The encoder design include three model: special code encoding, $3 \mathrm{~B} / 4 \mathrm{~B} \& 5 \mathrm{~B} / 6 \mathrm{~B}$ encoding, $\mathrm{RD}$ control. This three model make the $8 \mathrm{~B} / 10 \mathrm{~B}$ encoding characteristic, very clear and easy. As show in Figure.3.

Finished the process as below:

Firstly, you need to determine whether it is special character or not. If it is, there will be 12 kinds of code for special characters. Each character RD- and RD + shall be matched with different $10 \mathrm{~B}$ codes which is each inverted. According to the positive \& negative of RD and output it with the homologous value of code. Otherwise run codes of $3 \mathrm{~B} / 4 \mathrm{~B}$ and $5 \mathrm{~B} / 6 \mathrm{~B}[6]$.

The code of $3 \mathrm{~B} / 4 \mathrm{~B}$ and $5 \mathrm{~B} / 6 \mathrm{~B}$ according to the mapping rule of the code of $8 \mathrm{~B} / 10 \mathrm{~B}$, through the look-up table method, use the Verilog HDL language to achieve it, find the $4 \mathrm{~B}$ and $6 \mathrm{~B}$.

Let the $4 \mathrm{~B}$ and $6 \mathrm{~B}$ grouped to the $10 \mathrm{~B}$, and there are 4situations for this group: (1) when $4 \mathrm{~B}$ and $6 \mathrm{~B}$ are single value. (2) when $4 \mathrm{~B}$ is single value and $6 \mathrm{~B}$ is multiple-value. (3) when $4 \mathrm{~B}$ is multiple-value and $6 \mathrm{~B}$ is single value. (4) when $4 \mathrm{~B}$ and $6 \mathrm{~B}$ are all multiple-value[7].

Write the $8 \mathrm{~B} / 10 \mathrm{Bencoder}$ sequence by Verilog HDL language, the port of the encoder are made by 5 signals, there are clk, RSTn, kin, wr, dtin. Clk pin is the clock signal, RSTn pin is the reset signal, kin represent the dtin[7:0] special character input, wr is the enable signal[8].

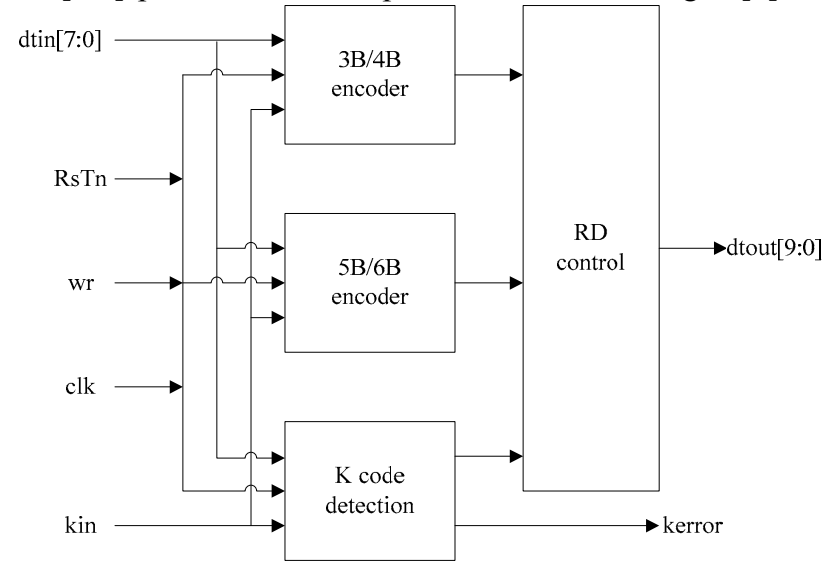

Figure 3. 8 B / 10 B code block diagram

\section{EXPERIMENTAL VERIFICATION}

In order to validate the effectiveness and feasibility of the above encoder design scheme. Based on high performance FPGA device, the simulation and integrated experiment is executed in the QuartusII9.0 integrated development. When kin is effective, the special characters streaming of illegal is input in dtin, the illegal mark signals Kerror will output effective high level which can be spotted in the simulation result, which illustrated the illegal code streaming was detected in the decoding circuit correctly. Decoding value 0101010101 was exported by dtout, simultaneously, which stated that decode can output by decoding circuit exactly, from the input and output of code streaming correspondence analysis, the problem of baseline migration and code streaming imbalance which appeared at optical fiber transmission data is solved by this plan very well. As shown in Figure.4. This program is suitable for integrated circuit and the FPGA design particularly, owing to its take less the FPGA internal resources, simple program structure, fast operation speed, low error code rate. Fully comply with the design requirements.

\section{CONCLUSION}

The design of $8 \mathrm{~B} / 10 \mathrm{~B}$ encoder is part of FPGA optical fiber communication, the demand of simple program logic relation, clear level demarcate, fast speed is put forward in the plan, which can be achieved by simulation. This program can be used in need $8 \mathrm{~B} / 10 \mathrm{~B}$ encoder of optical fiber coding remote data transmission system.

\section{ACKNOWLEDGMENT}

The subject is supported by Education Department of Henan Province Natural Science Research Projects Foundation (2010B460010) and Henan University of Science and Technology School Foundation (2009QN0020).

\section{REFERENCES}

[1] IBM Research and Development Journal, 1983,27(5).

[2] Using Actel FPGAs to Implement the 100Mbit/ s Ethernet Standard[J ]1 Actel , 1996, 5 (1) 1

[3] $8 \mathrm{~B} / 10 \mathrm{~B}[\mathrm{~J} / \mathrm{OL}]$, http://www.xilinx.com/cn/bvdocs/userguides/ug035.pdf.

[4] Cisco White Paper. Very Short Reach OC-192/STM-64 Interface: Optimizing for Network Intra-POP Interconnections [S].

[5] Widmer A X, Franaszek P A. A DC -Balance, Partitioned -Block 8B/10B Transmission Code [J]. IBM Journal of research and development, 1983, 23(5): 441-443.

[6] CAI Z, HAO J, TAN P H, et al . Efficient encoding of 1EEE802 . 1ln LDPC codes[J]. Electronics Letters, 2006 , 42(25): 1471-1472.

[7] GALLAGER RG. low density parity check codes[J]. IRE Trans. Inform. Theory, 1962(1): 21-28.

[8] PEREZ J M, FERNANDEZ V . 10w-cost encoding of IEEE 802. 11t[J]. Electronics Letters。2008, 44(4): 1411-1412.

[9] Adler R L , Coppersmith D , Hassner M1 Algorithms for Sliding Block C odes[J ]1 IEEE Trans. on Info. Theory, 1983 , IT- 29 :5 22.

[10] CYPRESS CY7B923 HOTLI NK Transmitter/ Receiver .CY7B923 HOT Link datasheet[ Z]. Cypress Semiconductor Corporation, 2003 


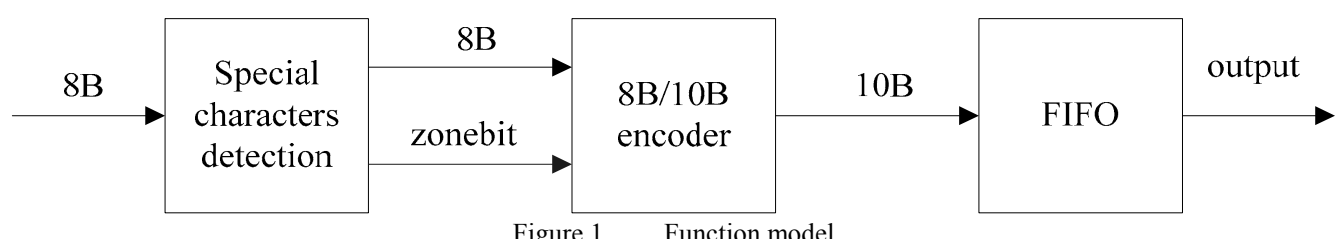

\begin{tabular}{|c|c|c|c|c|c|c|c|c|}
\hline 3B Decimal & $\mathbf{0}$ & $\mathbf{1}$ & $\mathbf{2}$ & $\mathbf{3}$ & $\mathbf{4}$ & $\mathbf{5}$ & $\mathbf{6}$ & $\mathbf{7}$ \\
\hline 3B binary(HGF) & 000 & 001 & 010 & 011 & 100 & 101 & 110 & 111 \\
& & & & & & & & \\
\hline 4B & $0100 /$ & 1001 & 0101 & $0011 /$ & $0010 /$ & 1010 & 0110 & $0001 / 1110 /$ \\
1100 & & 1101 & & & $1000 / 0111$ \\
\hline
\end{tabular}

TABLEII SPECIAL CODE ENCODING LIST

\begin{tabular}{|l|l|l|l|l|l|l|l|l|}
\hline $\begin{array}{l}\text { S.C. Byte } \\
\text { Name }\end{array}$ & \multicolumn{2}{|c|}{$\begin{array}{c}\text { S . C. Code } \\
\text { Name }\end{array}$} & HGF & EDCBA & abcdei & fghj & abcdei & fghj \\
\hline K28.0 & C0.0 & $(\mathrm{c} 00)$ & 000 & 00000 & 001111 & 0100 & 110000 & 1011 \\
\hline K28.1 & $\mathrm{C} 1.0$ & $(\mathrm{c} 01)$ & 000 & 00001 & 001111 & 1001 & 110000 & 0110 \\
\hline K28.2 & $\mathrm{C} 2.0$ & $(\mathrm{c} 02)$ & 000 & 00010 & 001111 & 0101 & 110000 & 1010 \\
\hline K28.3 & $\mathrm{C} 3.0$ & $(\mathrm{c} 03)$ & 000 & 00011 & 001111 & 0011 & 110000 & 1100 \\
\hline K28.4 & $\mathrm{C} 4.0$ & $(\mathrm{c} 04)$ & 000 & 00100 & 001111 & 0010 & 110000 & 1101 \\
\hline K28.5 & $\mathrm{C} 5.0$ & $(\mathrm{c} 05)$ & 000 & 00101 & 001111 & 1010 & 110000 & 0101 \\
\hline K28.6 & $\mathrm{C} 6.0$ & $(\mathrm{c} 06)$ & 000 & 00110 & 001111 & 0110 & 110000 & 1001 \\
\hline K28.7 & $\mathrm{C} 7.0$ & $(\mathrm{c} 07)$ & 000 & 00111 & 001111 & 1000 & 110000 & 0111 \\
\hline K23.7 & $\mathrm{C} 8.0$ & $(\mathrm{c} 08)$ & 000 & 01000 & 111010 & 1000 & 000101 & 0111 \\
\hline K27.7 & $\mathrm{C} 9.0$ & $(\mathrm{c} 09)$ & 000 & 01001 & 110110 & 1000 & 001001 & 0111 \\
\hline K29.7 & $\mathrm{C} 10.0$ & $(\mathrm{c} 0$ A) & 000 & 01010 & 101110 & 1000 & 010001 & 0111 \\
\hline K30.7 & $\mathrm{C} 11.0$ & $(\mathrm{c} 0 \mathrm{~B})$ & 000 & 01011 & 011110 & 1000 & 100001 & 0111 \\
\hline
\end{tabular}

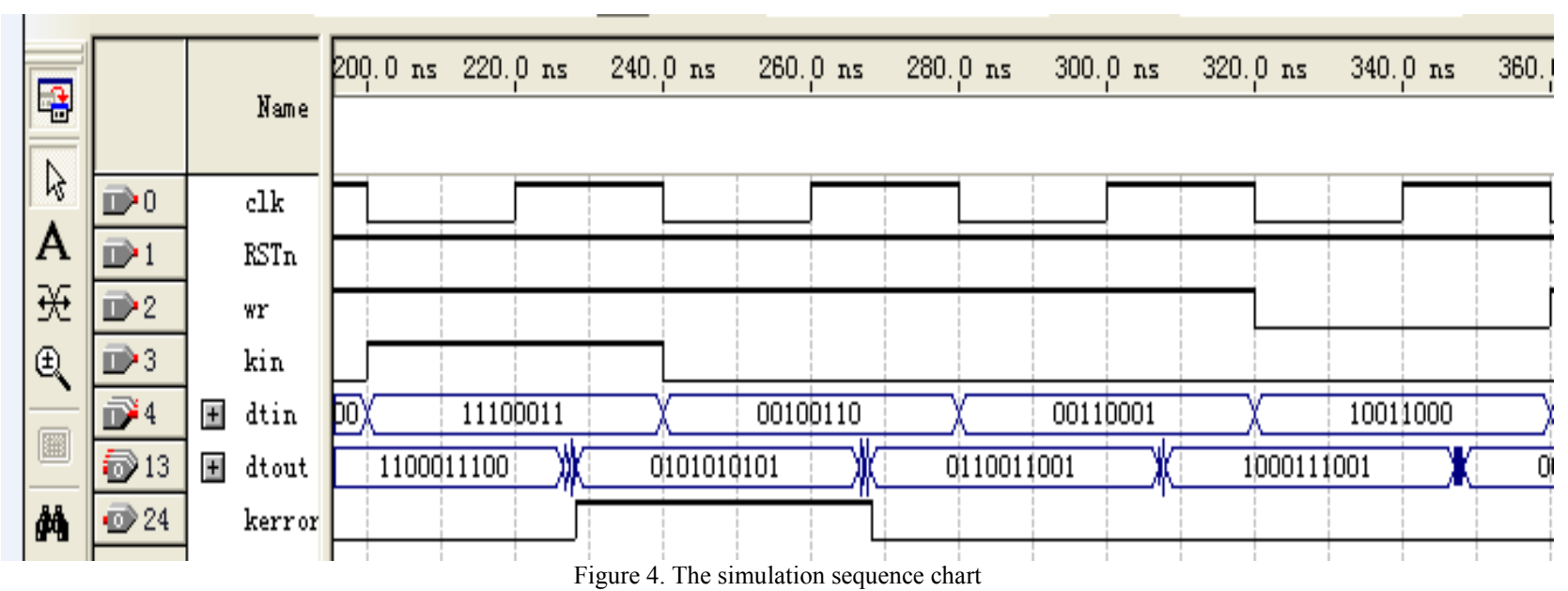

